American Journal of Infectious Diseases 7 (3): 61-66, 2011

ISSN 1553-6203

(C) 2011 Science Publications

\title{
Design, Synthesis, Biological Screening and Structure Activity Relationship Study of 4, 6-Dimethyl-2-(Substituted) Mercapto-3-(Substituted) Pyridines as Anti Tubercular Agents
}

\author{
${ }^{1}$ Mehul M. Patel and ${ }^{2}$ Mahesh T. Chhabriya \\ ${ }^{1}$ Department of Pharmaceutical Chemistry, Ramanbhai Patel College of Pharmacy, \\ Charotar University of Science and Technology, Changa 388421, India \\ ${ }^{2}$ Department of Pharmaceutical Chemistry, L.M. College of Pharmacy, \\ Gujarat University, India
}

\begin{abstract}
Problem statement: Tuberculosis is a leading global mortality factor which has not been effectively controlled, with 1.7 million deaths per year and 8.9 million new cases. Aerobic microbe Mycobacterium tuberculosis H37Rv (MTB) is the causative agent of tuberculosis. Although many active antitubercular agents have since been developed, drug resistance will continue to be a problem. Therefore, there is a clear need for the discovery of new derivatives with antitubercular activity for the management of tuberculosis. It was observed from the literature that pyridine nucleus have significant antitubercular activity. On the basis of that we have synthesized some new derivatives of pyridines and investigated their antimycobacterial properties. Approach: Two series of 3-cyano-4, 6-dimethyl-2(Alkyl/Aryllthio) pyridine and 4, 6-dimethyl-2-(Alkyl/Aryllthio) nicotinamide have been designed and synthesized from malanonitrile. The title compounds were evaluated at $12.5,25$ and $100 \mu \mathrm{g} \mathrm{mL}^{-1}$ concentrations for their anti tubercular activity against Mycobacterium tuberculosis $\mathrm{H}_{37} \mathrm{Rv}$ using Lowenstein Jensen method (proportion method). Results: Many of the synthesized compounds exhibit significant anti tubercular activity in comparison to Isoniazide while other compounds have shown promising ant tubercular activity. All the synthesized compounds screened for antimycobacterial activity were found significantly active against $M$. tuberculosis at the concentration $12.5 \mu \mathrm{g} \mathrm{mL}{ }^{-1}$. Conclusion: The antituberculosis screening data revealed that all the tested compounds $3 \mathrm{a}-3 \mathrm{~d}$ and $4 \mathrm{a}-4 \mathrm{~d}$ showed moderate to very good inhibitory activity. The compounds $4 \mathrm{a}-4 \mathrm{~d}$ showed very good antituberculosis activity. The good activity is attributed to the presence of substituted alkyl group at position-2 and amide group at position-3 of pyridine ring. Structure Activity Relationship (SAR) study reveals that with the increase in the chain length of alkyl group at position-2 has tremendously increased the activity of the title molecules.
\end{abstract}

Key words: Substituted pyridines, lowenstein jensen method, antitubercular activity, synthesized compounds, Mycobacterium tuberculosis, Acid Fast Bacillus (AFB)

\section{INTRODUCTION}

Tuberculosis is a characterized as a chronic bacterial infection caused by Mycobacterium tuberculosis, an aerobic Acid Fast Bacillus (AFB). TB is contagious and spreads through the air; if not treated properly, each person infects average 10-15 people every year. 2 billion people, equal to one third of the world's total population, are infected with TB bacilli. Two of every five person-more than 400 million have latent tuberculosis infection. Tuberculosis is currently the leading killer of the youth, women and AIDS patients throughout the world. Although many active antitubercular agents have since been developed, a disturbing co-occurrence with the use of present drugs as single agent has developed drug resistance (Swaminathan, 2002; Patel et al., 2007; Williams et al., 2002; Attaby et al., 1999; Rajni and Meena, 2011). The development of this resistance can be forestalled through the use of combination regimens, it is clear that drug resistance will continue to be a problem. Therefore, there is a clear need for the discovery of new derivatives with antitubercular activity for the management of tuberculosis. It was observed from the

Corresponding Author: Mehul M. Patel, Pharmaceutical Chemistry Department, Ramanbhai Patel College of Pharmacy, Charotar University of Science and Technology, At and po: Changa-388 421,

Dist-Anand, Gujarat, India Tel: 91-9879247492 Fax: 02697-247100 
literature that certain six member heterocyclic compounds possess interesting biological activity. Among them the compounds bearing pyridine nucleus have wide applications in medicinal chemistry. These compounds also have been reported to have significant antitubercular activity (Agrawal et al., 2007).

In view of these facts and in continuation of our studies on the synthesis of biologically active substituted pyridines, it was considered of interest to synthesize 2,3,4,6 tetra substituted pyridine compounds. For SAR study, alkyl substituent has been introduced at positions 2 to increase the lipophilicity. On the other hand, 3-cyano group has been replaced with the amide group. The title compounds thus synthesized were evaluated for their ant tubercular properties.

\section{MATERIALS AND METHODS}

Chemistry: The synthesis of 3-cyano-4, 6-dimethyl-2alkylthiopyridines and 4, 6-dimethyl-2-(alkylthio) nicotinamide from malanonitrile was performed as shown in Fig. 1. In the initial step, $\mathrm{H}_{2} \mathrm{~S}$ gas pass through Malanonitrile In the presence of triethylamine gave thiocyanocetamide which was further react with acetylacetone to gave Cyano-4, 6-dimethyl-2mercaptopyridine (2). The compounds 3a-3d were synthesized by reacting Cyano-4, 6-dimethyl-2mercaptopyridine (2) with alkyl/aryl halide. Compounds $4 \mathrm{a}-4 \mathrm{~d}$ was synthesized by acid catalyzed hydrolysis of compounds $3 \mathrm{a}-3 \mathrm{~d}$. The purity of the synthesized compounds was controlled by TLC. Spectral data like IR, ${ }^{1} \mathrm{H}$ NMR and mass of all the newly synthesized compounds were in full agreement with the proposed structures.

General: All the melting points were determined in open capillaries and are uncorrected. Thin layer chromatography was performed on microscopic slides $(2 \times 7.5 \mathrm{~cm} \mathrm{sec})$ coated with Silica-Gel-G and spots were visualized by exposure to iodine vapor. UV spectra were recorded in UV-VIS 160A Shimadzu spectrophotometer. IR spectra of all compounds were recorded in $\mathrm{KBr}$ on FT-IR 8400S Shimadzu spectrophotometer using KBr. Mass spectra were obtained using 2010EV LCMS Shimadzu instrument. ${ }^{1} \mathrm{H}$ and ${ }^{13} \mathrm{C}$ NMR spectra were obtained in $\mathrm{CDCl}_{3}$ on BRUKER Advance-II $400 \mathrm{MHZ}$ instrument and chemical shift were measured as parts per million downfield from Tetramethylsilane (TMS) as internal standard.

\section{Experimental section:}

Synthesis of thiocyanoacetamide (1) (Schmidth and Kubitzek, 1960): To a solution of malanonitrile $(6.6 \mathrm{~g}$, $0.1 \mathrm{moL})$ in ethanol $(20 \mathrm{~mL}), 0.5 \mathrm{~mL}$ of triethylamine was added. The mixture was continuous bubbled with $\mathrm{H}_{2} \mathrm{~S}$ gas for 3-4 h. with occasional shaking. Two crops of crystals were isolated. Recrystallized from ethanol, to yield $7.5 \mathrm{~g}(75 \% \mathrm{w} / \mathrm{w})$ of crystalline product. M.P. $115-116^{\circ} \mathrm{C}\left(116-117^{\circ} \mathrm{C}\right)$.

Synthesis of 3-Cyano-4,6-dimethyl-2mercaptopyridine (2) (Schmidth and Kubitzek, 1960): To a suspension of thiocyanoacetamide (10 g, 0.1 $\mathrm{mol})$ in ethanol $(100 \mathrm{~mL})$, a mixture of acetylacetone $(10$ $\mathrm{g}, \quad 0.1 \mathrm{~mol})$ and triethylamine $(1 \mathrm{~mL})$ was added dropwise with constant shaking. The mixture was allowed to stand at room temperature for $1 \mathrm{~h}$. Solid obtained was filtered under suction, dried and recrystallized from ethanol, to yield $12.49 \mathrm{~g}(76.21 \% \mathrm{w} / \mathrm{w})$ of crystalline product M.P. $260-261^{\circ} \mathrm{C}\left(264^{\circ} \mathrm{C}\right)$.

General procedure for the synthesis of 3-cyano-4,6dimethyl-2-alkylthiopyridine (3a-3d): 3-cyano-4,6dimethyl-2-alkylthiopyridines (3a-3d) were synthesized by alkylation of thiolate anion obtained by reaction of 3-cyano-4,6-dimethyl-2-mercaptopyridine (2) with sodium hydroxide as per Fig. 2.

To a cool solution of $20 \% \mathrm{w} / \mathrm{v} \mathrm{NaOH}(15 \mathrm{~mL}), 3-$ cyano-4, 6-dimethyl-2-mercaptopyridine (1.64 g, 0.01 mol) was added. The mixture was stirred at $5-10^{\circ} \mathrm{C}$. Tetrabutyl ammonium bromide was added as a phase transfer catalyst. Then Alkyl halide in ethanol $(0.1 \mathrm{~mol}$ in $10 \mathrm{~mL}$ ethanol) was added dropwise. After complete addition of, Alkyl halide, mixture was further stirred for 4-5 $\mathrm{h}$ and then kept overnight at room temperature.

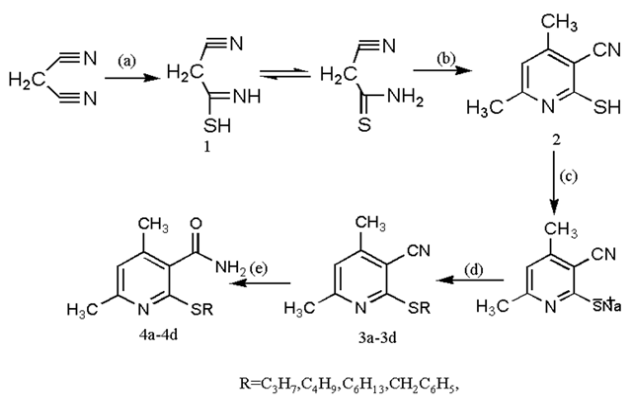

Fig. 1: (a) $\mathrm{H}_{2} \mathrm{~S}$,ethanol ,triethylamine (b) Acetyl acetone, ethanol ,triethylamine; (c) $\mathrm{NaOH}, 0$ $10{ }^{\circ} \mathrm{C} \quad(\mathrm{d})$ Alkyl halide (R-X), $\mathrm{R}=$ $\mathrm{C}_{3} \mathrm{H}_{7}, \mathrm{C}_{4} \mathrm{H}_{9}, \mathrm{C}_{6} \mathrm{H}_{13}, \mathrm{CH}_{2} \mathrm{C}_{6} \mathrm{H}_{5}, \quad \mathrm{X}=\mathrm{Cl}$ or $\mathrm{Br}$, TBAB(Tetra Butyl Ammonium Bromide); (e) $\mathrm{H}_{2} \mathrm{SO}_{4}(50 \%)$, reflux, 1-2h Thiocyanoacetamide (1), 3-Cyano-4,6-dimethyl-2-mercaptopyridine (2), 3-cyano-4,6-dimethyl-2-alkylthiopyridine $(3 \mathrm{a}-3 \mathrm{~d}), \quad \mathrm{R}=\mathrm{C}_{3} \mathrm{H}_{7}(3 \mathrm{a}), \quad \mathrm{C}_{4} \mathrm{H}_{9}(3 \mathrm{~b}), \quad \mathrm{C}_{6} \mathrm{H}_{13}(3 \mathrm{c})$, $\mathrm{CH}_{2} \mathrm{C}_{6} \mathrm{H}_{5}(3 \mathrm{~d})$ and 4,6-dimethyl-2(alkylthio)nicotinamide (4a-4d) $\mathrm{R}=\mathrm{C}_{3} \mathrm{H}_{7}(4 \mathrm{a})$, $\mathrm{C}_{4} \mathrm{H}_{9}(4 \mathrm{~b}), \mathrm{C}_{6} \mathrm{H}_{13}(4 \mathrm{c}), \mathrm{CH}_{2} \mathrm{C}_{6} \mathrm{H}_{5}(4 \mathrm{~d})$ 
Am. J. Infect. Dis., 7 (3): 61-66, 2011

Table 1: Physical characteristics of 3-cyano-4,6-dimethyl-2-alkylthiopyridine (3a-3d)

\begin{tabular}{|c|c|c|c|c|c|c|c|c|c|c|}
\hline Comp. No. & $\mathrm{R}$ & $\begin{array}{l}\text { Molecular } \\
\text { formulae }\end{array}$ & $\begin{array}{l}\text { Mol.wt } \\
\mathrm{g} / \mathrm{mol}\end{array}$ & B.P. $\left({ }^{\circ} \mathrm{C}\right)$ & M.P. $\left({ }^{\circ} \mathrm{C}\right)$ & $\begin{array}{l}\text { Yield } \\
(\% \mathrm{w} / \mathrm{w})\end{array}$ & $\begin{array}{l}\lambda_{\max } \\
(\mathrm{nm})\end{array}$ & Mass $(\mathrm{m} / \mathrm{e})$ & $\begin{array}{l}{ }^{1} \mathrm{H} \text { NMR } \\
(\delta \mathrm{ppm}, \mathrm{CDCl} 3)\end{array}$ & $\begin{array}{l}\text { I.R. } \\
\left(\mathrm{cm}^{-1}, \mathrm{KBr}\right)\end{array}$ \\
\hline$\overline{3 a}$ & $\mathrm{C}_{3} \mathrm{H}_{7}$ & $\mathrm{C}_{11} \mathrm{H}_{14} \mathrm{~N}_{2} \mathrm{~S}$ & 206 & $>300$ & --- & 61.16 & $\begin{array}{l}311.5 \\
270\end{array}$ & $\begin{array}{l}207(\mathrm{M}+1) \\
163(\mathrm{M}-43)\end{array}$ & --- & $\begin{array}{l}\text { 2964, 2922(C-H) } \\
2216(\mathrm{CN}) \\
1579(\mathrm{C}-\mathrm{N}) \\
879(\mathrm{C}-\mathrm{H} . \mathrm{pyr})\end{array}$ \\
\hline $3 b$ & $\mathrm{C}_{4} \mathrm{H}_{9}$ & $\mathrm{C}_{12} \mathrm{H}_{16} \mathrm{~N}_{2} \mathrm{~S}$ & 220 & $>300$ & --- & 81.81 & $\begin{array}{l}312 \\
270\end{array}$ & & $\begin{array}{l}1.45 \text { (sextet, } 2 \mathrm{H}, \mathrm{S} \\
\left.\left(\mathrm{CH}_{2}\right) 2 \mathrm{CH}_{2} \mathrm{CH}_{3}\right), \\
1.70\left(\text { quintet, } 2 \mathrm{H}, \mathrm{SCH}_{2}\right. \\
\left.\mathrm{CH}_{2} \mathrm{CH}_{2} \mathrm{CH}_{3}\right), \\
\text { 2.42(s,3H,-6CH } \\
\text { 3-pyridine), } \\
\text { 2.49(s,3H, } \\
\left.-4 \mathrm{CH}_{3} \text {-pyridine }\right), \\
\left.\text { 3.25(t,2H,SCH} \mathrm{CH}_{2} \mathrm{CH}_{2} \mathrm{CH}_{3}\right), \\
\text { 6.75(s,1H,pyridine proton) }\end{array}$ & $\begin{array}{l}2956,2929(\mathrm{C}-\mathrm{H}) \\
2217(\mathrm{CN}) \\
1579(\mathrm{C}-\mathrm{N}) \\
873(\mathrm{C}-\mathrm{H} \text { pyr })\end{array}$ \\
\hline $3 c$ & $\mathrm{C}_{6} \mathrm{H}_{13}$ & $\mathrm{C}_{14} \mathrm{H}_{20} \mathrm{~N}_{2} \mathrm{~S}$ & 248 & $>300$ & --- & 81.81 & 254 & $\begin{array}{l}249 \\
(\mathrm{M}+1) \\
163.2 \\
(\mathrm{M}-85)\end{array}$ & --- & $\begin{array}{l}2952,2929(\mathrm{C}-\mathrm{H}) \\
2216.06(\mathrm{C}-\mathrm{CN}) \\
1579(\mathrm{C}-\mathrm{N}) \\
873(\mathrm{C}-\mathrm{H} \text { pyr })\end{array}$ \\
\hline $3 d$ & $\mathrm{CH}_{2} \mathrm{C}_{6} \mathrm{H}_{5}$ & $\mathrm{C}_{15} \mathrm{H}_{14} \mathrm{~N}_{2} \mathrm{~S}$ & 254 & --- & $87-89$ & 55 & 244 & $255(\mathrm{M}+1)$ & --- & $\begin{array}{l}2979,2929(\mathrm{C}-\mathrm{H}) \\
2216.06(\mathrm{C}-\mathrm{CN}) \\
1587(\mathrm{C}-\mathrm{N}) \\
873(\mathrm{C}-\mathrm{H} \text { pyr })\end{array}$ \\
\hline
\end{tabular}

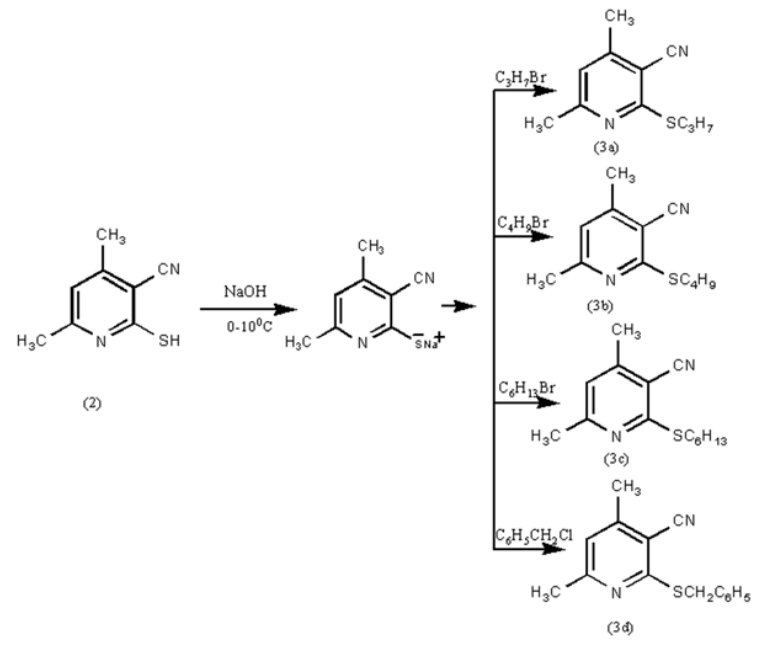

Fig. 2: Synthesis of 3-cyano-4, 6-dimethyl-2alkylthiopyridine (3a-3d)

Two layers were separated out, upper organic layered was collected and washed with water. Physical characteristics data of 3-cyano-4, 6dimethyl-2-alkylthiopyridine (3a-3d) were given in Table 1 .

Synthesis of 2-(propylthio)-4,6dimethylnicotinonitrile(3a): To a cool solution of 20\%w/v NaOH (15 mL), 3-cyano-4,6-dimethyl-2- mercaptopyridine $(1.64 \mathrm{~g}, 0.01 \mathrm{~mol})$ was added. The mixture was stirred at $5-10^{\circ} \mathrm{C}$. Tetrabutyl ammonium bromide was added as a phase transfer catalyst. Then Propyl bromide in ethanol $(0.1 \mathrm{~mol}$ in $10 \mathrm{~mL}$ ethanol) was added drop wise. After complete addition, mixture was further stirred for 4-5 h and then kept overnight at room temperature. Two layers were separated out, upper organic layered was collected and washed with water.

\section{Synthesis of 2-(butylthio)-4,6-} dimethylnicotinonitrile(3b): To a cool solution of $20 \% \mathrm{w} / \mathrm{v} \mathrm{NaOH}$ (15 mL), 3-cyano-4,6-dimethyl-2mercaptopyridine $(1.64 \mathrm{~g}, 0.01 \mathrm{~mol})$ was added. The mixture was stirred at $5-10^{\circ} \mathrm{C}$. Tetrabutyl ammonium bromide was added as a phase transfer catalyst. Then butyl bromide in ethanol $(1.0736 \mathrm{~mL}$ in $10 \mathrm{~mL}$ ethanol) was added drop wise. After complete addition of butyl bromide, mixture was further stirred for 4-5 $\mathrm{h}$ and then kept it overnight at room temperature. Two layers were separated out, upper organic layer was collected and washed with water, to yield $1.80 \mathrm{~g}(81.81 \% \mathrm{w} / \mathrm{w})$ product B.P. $>300^{\circ} \mathrm{C}$.

Synthesis of 2-(hexylthio)-4,6dimethylnicotinonitrile(3c): To a cool solution of 20\%w/v NaOH (15 mL), 3-cyano-4,6-dimethyl-2mercaptopyridine $(1.64 \mathrm{~g}, 0.01 \mathrm{~mol})$ was added. The mixture was stirred at $5-10^{\circ} \mathrm{C}$. Tetrabutyl ammonium bromide was added as a phase transfer catalyst. 
Am. J. Infect. Dis., 7 (3): 61-66, 2011

Table 2: Physical characteristics of 4, 6-dimethyl-2-(alkylthio) nicotinamide (4a-4d)

\begin{tabular}{|c|c|c|c|c|c|c|c|c|c|}
\hline Comp. No. & $\mathrm{R}$ & $\begin{array}{l}\text { Molecular } \\
\text { formulae }\end{array}$ & $\begin{array}{l}\text { Mol.wt } \\
\mathrm{g} / \mathrm{mol}\end{array}$ & M.P. $\left({ }^{\circ} \mathrm{C}\right)$ & $\begin{array}{l}\text { Yield } \\
(\% \text { w/w })\end{array}$ & $\begin{array}{l}\lambda_{\max } \\
(\mathrm{nm})\end{array}$ & $\begin{array}{l}\text { Mass } \\
(\mathrm{m} / \mathrm{e})\end{array}$ & $\begin{array}{l}1 \mathrm{H} \mathrm{NMR} \\
(\delta \mathrm{ppm}, \mathrm{CDCl} 3)\end{array}$ & $\begin{array}{r}\text { I.R. } \\
\left(\mathrm{cm}^{-1}, \mathrm{KBr}\right)\end{array}$ \\
\hline$\overline{4 a}$ & $\mathrm{C}_{3} \mathrm{H}_{7}$ & $\mathrm{C}_{11} \mathrm{H}_{16} \mathrm{~N}_{2} \mathrm{OS}$ & 224 & $146-148$ & 70.55 & $\begin{array}{l}293.5 \\
358\end{array}$ & & $\begin{array}{l}\left.\text { 1.01(t, } 3 \mathrm{H}, \mathrm{SCH}_{2} \mathrm{CH}_{2} \mathrm{CH}_{3}\right) \\
1.70\left(\mathrm{sextei}, 2 \mathrm{H}, \mathrm{SCH}_{2} \mathrm{CH}_{2} \mathrm{CH}_{3}\right) \\
2.29\left(\mathrm{~s}, 3 \mathrm{H},-4 \mathrm{CH}_{3} \text {-pyridine }\right) \\
2.44\left(\mathrm{~s}, 3 \mathrm{H},-6 \mathrm{CH}_{3}-\mathrm{pyridine}\right) \\
3.17\left(\mathrm{t}, 2 \mathrm{H}, \mathrm{SCH}_{2} \mathrm{CH}_{2} \mathrm{CH}_{3}\right) \\
5.85(\mathrm{~s}, 1 \mathrm{H}, \mathrm{C}=\mathrm{NH}) \\
6.13(\mathrm{~s}, 1 \mathrm{H}, \mathrm{C}-\mathrm{OH}) \\
6.70(\mathrm{~s}, 1 \mathrm{H}, \text { pyridine proton })\end{array}$ & $\begin{array}{l}\text { 3419.59(N-H) } \\
2929,2956(\mathrm{C}-\mathrm{H}) \\
1639(\mathrm{C}=\mathrm{O}) \\
1585.38(\mathrm{C}-\mathrm{N} \text { pyr }) \\
873(\mathrm{C}-\mathrm{H} \text { pyri })\end{array}$ \\
\hline $4 b$ & $\mathrm{C}_{4} \mathrm{H}_{9}$ & $\mathrm{C}_{12} \mathrm{H}_{18} \mathrm{~N}_{2} \mathrm{OS}$ & 238 & $140-142$ & 73.11 & $\begin{array}{l}293.5 \\
358\end{array}$ & $\begin{array}{l}239 \\
(\mathrm{M}+1) \\
221.9 \\
(\mathrm{M}-16)\end{array}$ & & $\begin{array}{l}\text { 3409.91(N-H), } \\
\text { 2929, 2954(C-H), } \\
\text { 1635(C = O), } \\
1585(\mathrm{C}-\mathrm{N} \text { pyr. }) \\
873(\mathrm{C}-\mathrm{H} \text { pyri. })\end{array}$ \\
\hline $4 c$ & $\mathrm{C}_{6} \mathrm{H}_{13}$ & $\mathrm{C}_{14} \mathrm{H}_{22} \mathrm{~N}_{2} \mathrm{OS}$ & 266 & $112-114$ & 80.64 & 254 & $\begin{array}{l}267 \\
(M+1) \\
250 \\
(M-16)\end{array}$ & & $\begin{array}{l}3417(\mathrm{~N}-\mathrm{H}) \\
2920,2956(\mathrm{C}-\mathrm{H}) \\
1643.24(\mathrm{C}=\mathrm{O}) \\
1587.31(\mathrm{C}-\mathrm{N} \text { pyr }) \\
873.6(\mathrm{C}-\mathrm{H} \text { pyri })\end{array}$ \\
\hline $4 d$ & $\mathrm{CH}_{2} \mathrm{C}_{6} \mathrm{I}$ & $\mathrm{C}_{15} \mathrm{H}_{16} \mathrm{~N}_{2} \mathrm{OS}$ & 272 & $132-134$ & 75.07 & 244 & & $\begin{array}{l}2.28\left(\mathrm{~s}, 3 \mathrm{H},-4 \mathrm{CH}_{3}-\text { pyridine }\right) \\
2.49\left(\mathrm{~s}, 3 \mathrm{H},-6 \mathrm{CH}_{3}-\text { pyridine }\right) \\
4.44\left(\mathrm{~s}, 2 \mathrm{H}, \mathrm{SCH}_{2} \mathrm{C}_{6} \mathrm{H}_{5}\right) \\
5.58(\mathrm{~s}, 1 \mathrm{H}, \mathrm{C}=\mathrm{NH}) \\
5.77(\mathrm{~s}, 1 \mathrm{H}, \mathrm{C}-\mathrm{OH}) \\
6.74(\mathrm{~s}, 1 \mathrm{H}, \text { pyridine proton }) \\
7.25(5 \mathrm{H}, \mathrm{Ar}-\mathrm{H})\end{array}$ & $\begin{array}{l}3369.41(\mathrm{~N}-\mathrm{H}) \\
2923,2974(\mathrm{C}-\mathrm{H}) \\
1639.38(\mathrm{C}=\mathrm{O}) \\
1581(\mathrm{C}-\mathrm{N} \text { pyr. } \\
871.7(\mathrm{C}-\mathrm{H} \text { pyri. })\end{array}$ \\
\hline
\end{tabular}

Then hexyl bromide in ethanol $(1.40 \mathrm{~mL}$ in $10 \mathrm{~mL}$ ethanol) was added dropwise. After complete addition of hexyl bromide, mixture was further stirred for 4-5 h and then kept it overnight at room temperature. Two layers were separated out, upper organic layer was collected and washed with water, to yield $1.35 \mathrm{~g}$ $(81.81 \% \mathrm{w} / \mathrm{w})$ product B.P. $>300^{\circ} \mathrm{C}$.

\begin{abstract}
Synthesis of 2-(benzylthio)-4,6dimethylnicotinonitrile (3d): To a cool solution of 20\% w/v NaOH (15 ml), 3-cyano-4,6-dimethyl-2mercaptopyridine (1.64 g, $0.01 \mathrm{~mol})$ was added. The mixture was stirred at $5-10^{\circ} \mathrm{C}$. Tetrabutyl ammonium bromide was added as a phase transfer catalyst. Then benzyl chloride in ethanol $(0.01$ mole in $10 \mathrm{~mL}$ ethanol) was added drop wise. After complete addition of benzyl chloride, mixture was further stirred for 4-5 $\mathrm{h}$ and then kept it overnight at room temperature. Solid obtained was collected and washed with water, to yield $1.394 \mathrm{~g}$ $\left(55 \%\right.$ w/w) product. M.P. $87-89^{\circ} \mathrm{C}\left(87^{\circ} \mathrm{C}\right)$.
\end{abstract}

General procedure for the synthesis of 4,6-dimethyl2-(alkylthio)nicotinamide (4a-4d): Acid catalyzed hydrolysis of 4,6-dimethyl-2-(alkylthio)nicotinonitrile (3a-3d) under controlled temperature yielded 4,6- dimethyl-2-(alkylthio)nicotinamide (4a-ad). Acid catalyzed hydrolysis gave promising yield in 2thioalkyl derivatives as per Fig. 3 .

Figure 2 Synthesis of 4,6-dimethyl-2(alkylthio)nicotinamide (4a-4d):

$$
\begin{aligned}
& \mathrm{R}=\mathrm{C}_{3} \mathrm{H}_{7}(3 \mathrm{a}), \mathrm{C}_{4} \mathrm{H}_{9}(3 \mathrm{~b}), \mathrm{C}_{6} \mathrm{H}_{13}(3 \mathrm{c}), \mathrm{CH}_{2} \mathrm{C}_{6} \mathrm{H}_{5}(3 \mathrm{~d}) \\
& \mathrm{R}=\mathrm{C}_{3} \mathrm{H}_{7}(4 \mathrm{a}), \mathrm{C}_{4} \mathrm{H}_{9}(4 \mathrm{~b}), \mathrm{C}_{6} \mathrm{H}_{13}(4 \mathrm{c}), \mathrm{CH}_{2} \mathrm{C}_{6} \mathrm{H}_{5}(4 \mathrm{~d})
\end{aligned}
$$

3-Cyano-4, 6-dimethyl-2-(alkyllthio)pyridine (1.96 $\mathrm{g}, 0.0095 \mathrm{~mol}$ ) was added to $12 \mathrm{ml}$ of $50 \% \mathrm{v} / \mathrm{v} \mathrm{H}_{2} \mathrm{SO}_{4}$ and reflux for 1-2 h. The reaction mixture was brought to room temperature and neutralized by adding $20 \%$ w/v $\mathrm{NaOH}$ solution till basic to litmus. The solid obtained was filtered under suction, dried at room temperature and recrystallized from benzene. Physical characteristics data of 4, 6-dimethyl-2(alkylthio)nicotinamide (4a-4d) were given in Table 2.

Synthesis of 2-(propylthio)-4,6-dimethylnicotinamide (4a): 2-(propylthio)-4,6-dimethylnicotinonitrile (1.96 g, $0.0089 \mathrm{~mol})$ was added to $12 \mathrm{~mL}$ of $50 \%$ $\mathrm{v} / \mathrm{v} \quad \mathrm{H}_{2} \mathrm{SO}_{4}$ and reflux for $1-2 \mathrm{~h}$ at $100^{\circ} \mathrm{C}$. 
Am. J. Infect. Dis., 7 (3): 61-66, 2011

Table 3: Comparision of screened compound for activity against M.Tuberculosis with reference to isoniazide

\begin{tabular}{llll}
\hline Compound & Concentration $\left(12.5 \mu \mathrm{g} \mathrm{m}^{-1}\right)$ & Concentration $\left(25 \mu \mathrm{g} \mathrm{m}^{-1}\right)$ & Concentration $(100 \mu \mathrm{g} \mathrm{mL})$ \\
\hline Isoniazide $(1 \mu \mathrm{g} \mathrm{mL})$ & & ++++ & ++++ \\
3a & ++ & +++ & ++++ \\
3b & ++ & +++ & ++++ \\
3c & +++ & ++++ & ++++ \\
3d & +++ & +++ & ++++ \\
$4 \mathrm{a}$ & +++ & +++ & +++ \\
$4 \mathrm{~b}$ & +++ & +++ & ++++ \\
$4 \mathrm{c}$ & ++++ & ++++ & ++++ \\
$4 \mathrm{~d}$ & +++ & ++++ & ++ \\
\hline
\end{tabular}

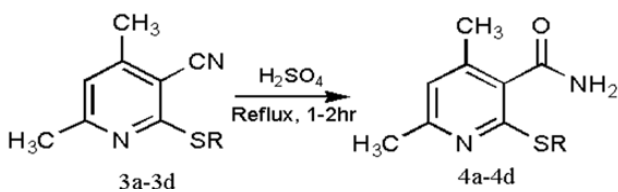

Fig. 3: Synthesis of 4,6-dimethyl-2(alkylthio)nicotinamide (4a-4d)

The reaction mixture was brought to room temperature and neutralized by adding $20 \% \mathrm{w} / \mathrm{v} \mathrm{NaOH}$ solution till basic to litmus. The precipitate obtained was filtered under suction, dried at room temperature and recrystallized from benzene, to yield $1.55 \mathrm{~g}$ $(73.11 \% \mathrm{w} / \mathrm{w})$ of crystalline product M.P. $140-142^{\circ} \mathrm{C}$.

Synthesis of 2-(butylthio)-4,6-dimethylnicotinamide (4b): 2-(butylthio)-4,6-dimethylnicotinonitrile (1.96 g, $0.0089 \mathrm{~mol}$ ) was added to $12 \mathrm{ml}$ of $50 \% \mathrm{v} / \mathrm{v}$ $\mathrm{H}_{2} \mathrm{SO}_{4}$ and reflux for $1-2 \mathrm{~h}$ at $100^{\circ} \mathrm{C}$. The reaction mixture was brought to room temperature and neutralized by adding $20 \% \mathrm{w} / \mathrm{v} \mathrm{NaOH}$ solution till basic to litmus. The precipitate obtained was filtered under suction, dried at room temperature and recrystallized from benzene, to yield $1.55 \mathrm{~g}$ $(73.11 \% \mathrm{w} / \mathrm{w})$ of crystalline product M.P. 140$142^{\circ} \mathrm{C}$.

\section{Synthesis of 2-(hexylthio)-4,6- dimethylnicotinamide(4c): 2-(hexylthio)-4,6-} dimethylnicotinonitrile $(1.96 \mathrm{~g}, 0.0079 \mathrm{moL})$ was added to $12 \mathrm{~mL}$ of $50 \% \mathrm{v} / \mathrm{v} \mathrm{H}_{2} \mathrm{SO}_{4}$ and reflux for $1-2 \mathrm{~h}$. The reaction mixture was brought to room temperature and neutralized by adding $20 \% \mathrm{w} / \mathrm{v} \mathrm{NaOH}$ solution till basic to litmus. The precipitate obtained was filtered under suction, dried at room temperature and recrystallized from benzene, to yield $2.54 \mathrm{~g}$ $\left(80.64 \%\right.$ w/w) of crystalline product. M.P. $112-114^{\circ} \mathrm{C}$

Synthesis of 2-(benzylthio)-4,6dimethylnicotinonitrile (4d) (Zawisza and Malinka, 1986): To a cool solution of $20 \% \mathrm{w} / \mathrm{v} \mathrm{NaOH}(15 \mathrm{~mL})$, 3-cyano-4,6-dimethyl-2-mercaptopyridine ( $1.64 \mathrm{~g}, 0.01$ mol) was added. The mixture was stirred at $5-10^{\circ} \mathrm{C}$.
Tetrabutyl ammonium bromide was added as a phase transfer catalyst. Then benzyl chloride in ethanol $(1.153 \mathrm{ml}$ in $10 \mathrm{~mL}$ ethanol) was added drop wise. After complete addition of benzyl chloride, mixture was further stirred for $4-5 \mathrm{~h}$ and then kept it overnight at room temperature. Solid obtained was collected and washed with water, to yield $1.394 \mathrm{~g}(55 \% \mathrm{w} / \mathrm{w})$ product. M.P. $87-89^{\circ} \mathrm{C}\left(87^{\circ} \mathrm{C}\right)$.

Antituberculosis activity by Lowenstein Jensen assay method: M. tuberculosis $\mathrm{H} 37 \mathrm{Rv}$ was grown in Lowenstein Jensen media. The culture was diluted to McFarland 1 standard with the same medium. From this, $50 \mathrm{~mL}$ of this culture were added to $15 \mathrm{~mL}$ of fresh medium in McCartney tubes. Stock solutions (1 $\mathrm{mg} \mathrm{mL} \mathrm{m}^{-1}$ ) of the test compounds were prepared in dimethyl sulphoxide (DMSO). The compounds were tested at $12.5,25$ and $100 \mu \mathrm{g} \mathrm{mL}^{-1}$ concentrations. Control wells had the same volumes of DMSO without any compound. Isoniazid $\left(1 \mu \mathrm{g} \mathrm{mL}^{-1}\right)$ served as positive control. After incubation at $37^{\circ} \mathrm{C}$ for 7 th week, it was found that all the McCartney tubes containing different dilutions of the test compounds did not show any type of growth of the mycobacteria as compared to control (isoniazide $1 \mu \mathrm{g} \mathrm{mL}^{-1}$ ). This was further confirmed by the Ziehl-Neilson staining of all sample, which shown absence of mycobacteria.

\section{RESULTS}

\section{Pharmacolgy:}

Antitubercular activity: The encouraging results from the antibacterial studies impelled us to go for the preliminary screening of the title compounds for their in vitro antituberculosis activity. The compounds were evaluated against Mycobacterium tuberculosis H37Rv ATCC 27294 strain using Lowenstein Jensen method; the observed MICs are presented in Table 3. Isoniazid (INH) $\left(1 \mu \mathrm{g} \mathrm{mL} L^{-1}\right)$ was used as standard drugs. The antituberculosis screening data revealed that all the tested compounds $3 \mathrm{a}-3 \mathrm{~d}$ and $4 \mathrm{a}-4 \mathrm{~d}$ showed moderate to very good inhibitory activity. The compounds $4 \mathrm{a}-4 \mathrm{~d}$ showed very good antituberculosis activity. 


\section{DISCUSSION}

The good activity is attributed to the presence of substituted alkyl group at position-2 and amide group at position -3 of pyridine ring. Structure Activity Relationship (SAR) study reveals that with the increase in the chain length of alkyl group at position- 2 has tremendously increased the activity of the title molecules.

3-cyano-4,6-dimethyl-2-alkylthiopyridine (3a-3d), $\mathrm{R}=\mathrm{C}_{3} \mathrm{H}_{7}(3 \mathrm{a}), \mathrm{C}_{4} \mathrm{H}_{9}(3 \mathrm{~b}), \mathrm{C}_{6} \mathrm{H}_{13}(3 \mathrm{c}), \mathrm{CH}_{2} \mathrm{C}_{6} \mathrm{H}_{5}(3 \mathrm{~d}) .4,6-$ dimethyl-2-(alkylthio)nicotinamide $(4 \mathrm{a}-4 \mathrm{~d}), \quad \mathrm{R}=$ $\mathrm{C}_{3} \mathrm{H}_{7}(4 \mathrm{a}), \mathrm{C}_{4} \mathrm{H}_{9}(4 \mathrm{~b}), \mathrm{C}_{6} \mathrm{H}_{13}(4 \mathrm{c}), \mathrm{CH}_{2} \mathrm{C}_{6} \mathrm{H}_{5}(4 \mathrm{~d})$.

\section{CONCLUSION}

The present study reports the successful synthesis, antibacterial and antituberculosis studies of a new class of 4, 6-dimethyl-2-(substituted) mercapto-3(substituted) pyridines carrying biologically active groups. Their screening results revealed that all the compounds showed moderate to very good activity against pathogenic strains. Study of structure-activity relationship showed that the presence of lipophilic group at position- 2 and the existence of amide at position- 3 are responsible for increased antituberculosis activity of the newly synthesizedcompounds. It can be concluded that 2-(alkyl) mercapto-3-(amido) pyridine derivatives has antituberculosis effect. All the compounds are found to be active against Mycobacterium tuberculosis at $12.5 \mu \mathrm{g}$ $\mathrm{mL}^{-1}$ concentration.

\section{ACKNOWLEDGEMENT}

The researchers are thankful to authorities of L. M. College of Pharmacy, Ahmedabad for providing necessary facilities and especially thankful to Dr. (Mrs.) Madhulika A. Mistry, Pathologist and Bacteriologist. K. J. Mehta of T. B. Hospital, Amargadh, Bhavnagar for antimycobacterial evaluations. Authors are also thankful to Punjab University, Chandigarh and Oxygen Healthcare Ltd., Ahmedabad for providing ${ }^{1} \mathrm{H}$ NMR and GC-MS spectral analyses of the compounds.

\section{REFERENCES}

Attaby, F.A., M.A. Elneairy and M.S. Elsayed, 1999. Synthesis and antimicrobial evaluation of new pyridine, thienopyridine and pyridothienopyrazole derivatives. Arch. Pharm. Res., 22: 194-201. PMID: 10230512

Patel, R.J., G.C. Patel, M.M. Patel and N.J. Patel, 2007. HIV Infection and Tuberculosis. Indian J. Pharm. Edu. Res., 41: 95-101.

Rajni and L.S. Meena, 2011. Unique characteristic features of Mycobacterium tuberculosis in relation to immune system. Am. J. Immunol., 7: 1-8. DOI: 10.3844/ajisp.2011.1.8

Schmidth, U. and H. Kubitzek, 1960. Synthesen mit den Thioamiden der Malonsäure, II. Thiopyridone aus Cyan-thioacetamid. Chem. Ber., 93: 15591565. DOI: $10.1002 /$ cber. 19600930716

Swaminathan, S., 2002. Clinical presentation and treatment of HIV-TB. Indian J. Tub., 49: 11-16.

Williams, D.A., W.O. Foye, T.L. Lemke, 2002. Foye's Principles of Medicinal Chemistry. 5th Edn., Lippincott Williams and Wilkins, Philadelphia, ISBN: 9780683307375, pp: 1114.

Zawisza, T. and W. Malinka, 1986. Synthesis and properties of some derivatives of $2 \mathrm{H}-4,6-$ dimethylpyrido[3,2-d]isothiazolin-3-one-1,1-

dioxide. Farmaco Sci., 41: 676-683. PMID: 3491004 